\title{
The critical-state yield stress (termination locus) of adhesive powders from a single numerical experiment
}

\author{
Stefan Luding • Fernando Alonso-Marroquín
}

Received: 15 March 2010 / Published online: 18 January 2011

(c) Springer-Verlag 2011

\begin{abstract}
Dry granular materials in a split-bottom ring shear cell geometry show wide shear bands under slow, quasistatic, large deformation. This system is studied in the presence of contact adhesion, using the discrete element method (DEM). Several continuum fields like the density, the deformation gradient and the stress tensor are computed locally and are analyzed with the goal to formulate objective constitutive relations for the flow behavior of cohesive powders. From a single simulation only, by applying time- and (local) space-averaging, and focusing on the regions of the system that experienced considerable deformations, the critical-state yield stress (termination locus) can be obtained. It is close to linear, for non-cohesive granular materials, and nonlinear with peculiar pressure dependence, for adhesive powdersdue to the nonlinear dependence of the contact adhesion on the confining forces. The contact model is simplified and possibly will need refinements and additional effects in order to resemble realistic powders. However, the promising method of how to obtain a critical-state yield stress from a single numerical test of one material is generally applicable and waits for calibration and validation.
\end{abstract}

Keywords Granular materials - Split-bottom shear bands . Inter-particle adhesive forces

S. Luding

Multi Scale Mechanics (MSM), CTW, UTwente, PO Box 217,

7500 AE, Enschede, The Netherlands

e-mail: s.luding@utwente.nl

F. Alonso-Marroquín ( $\varangle)$

School of Civil Engineering, The University of Sydney, Sydney, NSW 2006, Australia

e-mail: fernando.alonso@usyd.edu.au

\section{Introduction}

Granular materials are a paradigm of complex systems, where phenomena like segregation, clustering, arching, and shear-band formation occur due to the collective behavior of many particles interacting via contact forces. For geotechnical applications and industrial design in mechanicaland process-engineering, one of the main challenges is to obtain (macroscopic) continuum constitutive relations that allow to predict the flow behavior of the particles. The relevant macroscopic parameters are usually obtained from experimental and numerical tests on representative samples, so-called element-tests. As an example, as the basis for silodesign, the Jenike procedure to measure the so-called "incipient yield locus" (named yield locus in the following) is discussed in classical publications and textbooks, see e.g. Refs. [1-3].

DEM simulations of similar element-tests offer advantages with respect to physical experiments, as they provide more detailed information on forces and displacements at the grain scale. DEM allows the specification of particle properties and interaction laws and then the numerical solution of Newton's equations of motion $[4,5]$ of all particles. Finding the connection between the micro-mechanical properties and the macroscopic behavior involves the so-called micromacro transition [6-11]. Extensive "microscopic" simulations of many homogeneous small samples, i.e., so-called representative volume elements (RVE), have to be used to derive the macroscopic constitutive relations needed to describe the material within the framework of a continuum theory. In this study, however, the critical-state yield stresses at various pressure levels (which manifest the termination locus, or also called: critical-state locus or criticalstate yield surface) are obtained from a single numerical experiment. 
In this first introduction section, we review the different terminologies used in various fields concerning the phrases yield stress and locus, discuss their relation and theoretical interpretation, give some practical examples, and discuss the present approach. Section 2 is dedicated to the contact model, which contains normal elasto-plastic, viscous, and most important, adhesive forces (with artificially small friction in the tangential direction). In Sect. 3, the results from samples with different adhesion intensity are presented in the form of the termination locus. Finally, in Sect. 4, a short summary, conclusions, and an outlook are given.

\subsection{Different yield stresses and concepts}

To clarify the terminology, we review different concepts related to the material behavior and its measurementespecially concerning the yield of granular materials, see e.g., chapter 6 in the book by Nedderman [3].

The state of a granular material (e.g. in a shear cell) is usually characterized by some normal stress $\sigma$, the shear stress $\tau$, and the void fraction $\varepsilon=1-v$ (where $\varepsilon$ is the volume of voids divided by total volume and the volume fraction (density), $v$, is the volume of particles divided by total volume, i.e., the dimensionless density).

To determine the failure properties of the material, a normal stress $\sigma_{0}$ is applied and then the sample is sheared (in a given experimental set-up, which determines which stresses can be measured, e.g., as force divided by wall-area). As the shear stress increases the sample can dilate, however, this depends on the initial state of the material: one can observe over-, under- and critically consolidated behavior for initially high, low and intermediate densities, respectively. In many situations and for many materials, the stress reaches a maximum $\tau_{0}$ with void fraction $\varepsilon_{0}$ (density $\nu_{0}$ ). The point in stress space, $\left(\sigma_{0}, \tau_{0}\right)$ is called a yield stress or yield point of the material if the stress does not increase further. (This does not imply reversibility below and purely plastic behavior at yield, as some elasto-plastic models would). Note that the yield point depends sensibly on the history of the sample and, in many experimental configurations, the state of stress is not homogeneous throughout the sample. When sheared for larger strains, typically, the sample reaches a critical-state, where the measured values do not change anymore and (as paradigm) do not depend on the history of the sample. If this state is reproducibly reached, it can be used as starting (termination) point to obtain the yield locus of the material and we denote this termination point stress values by a star, see Fig. 1.

The concept of yield point (stress) is used in particle technology in a context different from engineering mechanics. In the latter case a yield point belongs to a yield surface that encloses a hypothetical region in stress space where only elastic deformations are possible. In the case of particulate

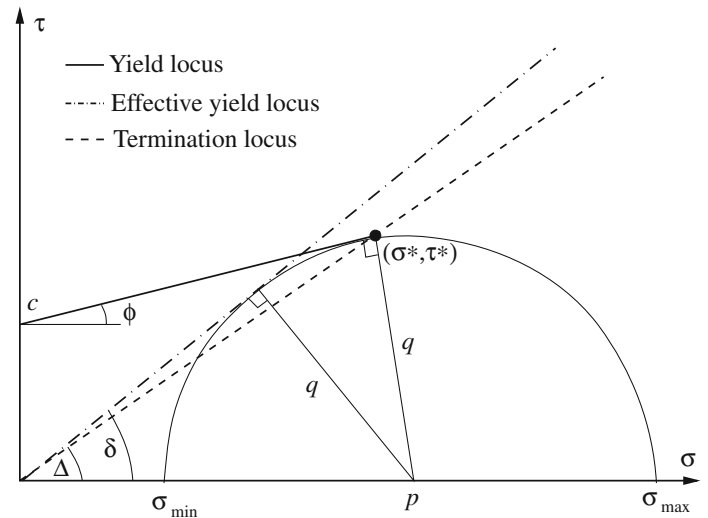

Fig. 1 Geometrical representation of the (incipient) yield locus, the effective yield locus and the termination locus, in the shear versus normal stress plane, according to Nedderman [3]. The so-called Mohrcircle represents the stress tensor with eigen-values $\sigma_{\min }$ and $\sigma_{\max }$, pressure $p$ and deviatoric stress $q$, as defined in the main text

materials there is no sound evidence of a purely elastic regime. Therefore we avoid here to introduce the concept of yield surface. Instead we refer here to the yield locus as the equivalent to the failure surface in engineering materials, i.e., the surface in the stress space that encloses all admissible stress states.

In powder technology, a very particular method is used to measure the (incipient) yield locus. The material sample must be first consolidated and then sheared under some constant load, $\sigma$, until the shear stress, $\tau$, has reached a constant (i.e., the critical-state with $\sigma^{*} \approx \sigma_{0}$ and $\tau^{*} \approx \tau_{0}$ ). Then the shear stress is released, i.e., the direction of shear must be reversed until the shear stress reduces almost to zero. With either the same or, usually, a lower load, shear is applied in the original direction until failure (yield) occurs. The peak shear stress measured this way represents one point on the yield locus in the shear versus normal stress representation, see Fig. 1. Reconsolidating with the same initial normal load, and following the same procedure of releasing and shear failure with a different load, produces further points on the yield locus that belongs to $\sigma_{0}$ with a consolidation density $\nu_{0}$ (void fraction $\varepsilon_{0}$ ).

For different consolidation normal stress, $\sigma_{1}$, another void fraction $\varepsilon_{1}$ will establish and, following the same procedure, another yield locus will be obtained. Different yield loci are often similar in shape and can be scaled using their end-point (termination) as reference, see Refs. [1,3].

If shear is applied further, beyond failure (yield), the shear stress usually decreases and saturates at a level that is smaller and independent of the consolidation stress, which is called sustained yield locus, critical-state yield stress, or termination locus, see Fig. 1. In contrast, if the load would be increased after consolidation, the powder would compact, i.e., the density would increase from its consolidation value 
and yield would take place on the so-called consolidation locus (not shown here, see Ref. [3]).

Note that the density at failure normally can not be measured experimentally, since the system is inhomogeneous, with a localized shear-band, where dilation takes place, while the density changes little outside of the shear-band. For the critical-state yield stress (termination locus), it is assumed that the density reaches an equilibrium value at large strain, which is close to the value defined by the consolidation density at the same stress-level. However, due to localized strain in the shear-band, this is not trivial in most geometries.

\subsection{Theoretical interpretation}

There is no fundamental reason that the yield loci be geometrically similar neither that they and the termination locus are straight lines. Nedderman names those materials "simple materials" [3], and Molerus [2] based his theory on the assumptions that (i) the adhesion forces at contact are proportional to the external stresses-which is not true for the contact model used in this study - and (ii) the (directional) probability distribution of adhesive forces is the same as the orientational distribution of the normal forces (which is subject to further study).

For simple materials, the yield locus is close to a line as shown in Fig. 1, and the yield locus is predicted according to Coulomb as:

$\tau=c+\mu_{m} \sigma$,

with the macroscopic coefficient of friction, $\mu_{m}=\tan \phi$, the internal angle of friction $\phi$, and the macroscopic cohesion $c$. If the yield locus is not straight, one still can apply linear fits in some point, e.g., at termination, or at some range of stresses, to obtain the effective values, $\phi_{e}$, and $c_{e}$. The (straight) termination locus is classified by the angle $\Delta$, so that $\tau^{*}=\mu_{m}^{*} \sigma^{*}=\tan \Delta \sigma^{*}$, where the stars denote the termination locus stresses shown in Fig. 1. The effective yield locus is the tangent to the same Mohr circle, which also goes through the origin with angle $\delta$ to the normal stress axis, so that

$\sin \delta=q / p$,

with the isotropic stress $p$ and the deviatoric stress $q=$ $\left(\sigma_{\max }-\sigma_{\min }\right) / 2$, which is based on the maximal and minimal eigenvalues of stress [11,12] (which are usually not known when only one normal and one shear stress can be measured). Note that $p$ is objective and well-defined (but not always available). This is in contrast to $\sigma^{*}$, which depends on the experimental configuration and the details of the measurement. In general, one has $p \neq \sigma^{*}$ and $q \neq \tau^{*}$ even though the values are close [12].

An alternative definition of the deviatoric stress is based on the second invariant of the stress tensor and is thus objective: $\sigma_{D}=\sqrt{I_{2} / 2}$, see Ref. [12]. For a stress tensor with intermediate eigenvalue $\sigma_{2}=\left(\sigma_{\max }+\sigma_{\min }\right) / 2$, which is colinear (coaxial) with the strain-rate in the critical-state, the three deviatoric stresses would be identical $\tau^{*}=q=\sigma_{D}$. Deviations from this identity were reported to be rather small [12], however, this is subject to further study. ${ }^{1}$

For the same geometry as used in this study, i.e., the split-bottom ring shear cell, the shear stress at critical state was measured for cohesionless materials with and without friction [11,12], and observed to be almost linear, being somewhat smaller for lower confining stress. This allowed to specify the macroscopic coefficient of friction, $\mu_{m}^{*} \neq \mu$, where $\mu$ is the coefficient of friction at contact. The deviator stress, $\sigma_{D}$ is slightly larger than the shear stress $\tau^{*}$ and the stress difference $q$ (unpublished data-not shown).

The internal angle of friction, $\phi$, represents the bulk friction of the static, consolidated material, while the effective angle of friction, $\delta$, is relevant for flowing materials. Only when the stress and strain rate are co-linear in the critical flow regime, the simple relation $\sin \delta=\tan \Delta$, which relates the angles of the effective and the termination locus, can be expected to hold. A more detailed study of co-linearity, as connected to the difference between shear and deviatoric stress, is far beyond the scope of this study with the focus on the termination locus from DEM simulations of particles with nonlinear contact adhesion.

\subsection{Practical examples}

In mechanical and chemical engineering, e.g., for the design of silos, the Jenike procedure $[3,13]$ is based on the knowledge of several yield loci, including also yield loci under time-consolidation, which tend to show greater shear stresses and cohesion than for instantaneous measurements. The flow-function of Jenikes procedure is based on the unconfined yield stress, $\sigma_{c}$, which can be obtained from the Mohr circle that goes through zero confining stress and is tangent to the yield locus. Different yield loci thus correspond to different $\sigma_{c}$. For further details we refer to the broad literature on this subject, since the unconfined yield stress is not directly related to the critical-state yield stress (termination locus) as discussed in this study. As will be shown, the termination locus is not a straight line for our material, which thus is not "simple".

In soil mechanics, there is something referred to as a "tension cut-off", see chapter 11 in Ref. [14], and applies to the case of overconsolidated (pre-stressed) clays. In this context, let us just call them stiff clays and ignore their history. Stiff clays behave in a similar manner to dense sands in that they expand when sheared and exhibit strain softening

\footnotetext{
$\overline{1}$ By definition, two tensors are called colinear, or coaxial, when the principal axes of both tensors coincide.
} 
behavior. Whether they exhibit a "cohesion intercept" on a Mohr-Coulomb diagram or not is still an open question due to the difficulty of performing reliable element tests in the laboratory at very low stresses. Some type of tension cut-off, resulting from simulations, will be shown in this paper, see Fig. 5.

In rheology, the yield stress of a sheared sample of material is the typical subject of investigation, see as mere examples, Refs. [15-19] and references therein. The role of the microstructure that can lead to effects like aging (or re-juvenation [20]), as well as concepts like shear-thinning or -thickening and their relation to the present study are not detailed further.

Also a discussion of the influence of different boundary conditions (uni- vs. bi-axial deformation or simple shear vs. pure shear) goes beyond the scope of this study, as does the discussion of different materials with more complex microstructure than particle systems (like e.g. polymers).

\subsection{Discussion of the present approach}

An alternative to a series of element tests is presented in this study. One can simulate an inhomogeneous geometry, where static regions co-exist with dynamic, flowing zones and, respectively, high density co-exists with dilated zonesat various pressure levels. From adequate local averaging over equivalent volumes-inside which all particles behave similarly - one can obtain from a single simulation already constitutive relations in a certain parameter range, as was done systematically in two-dimensional (2D) Couette ring shear cells $[9,21]$ and three-dimensional (3D) split-bottom ring shear cells $[11,12]$. The fact that the split-bottom shear cell has a free surface allows to scan a range of confining pressures between zero and $\sigma_{z z}^{\max }$, which is due to the weight of the material and determined by the filling height.

One special property of the split-bottom ring shear cell is the fact that the (wide) shear band is initiated at the bottom gap between the moving, outer and fixed, inner part of the bottom-wall and thus remains in many cases far away from the walls. The velocity field is well approximated by an errorfunction [11,12,22-24] with a width considerably increasing from bottom to top (free surface) [19,24-26]. The width of the shear-band is considerably larger than only a few particle diameters, as reported in many other systems. Effects like segregation in this system are not discussed here, instead we refer to a recent study [27] and the references therein.

The micro-macro data-analysis of the simulations provides data-sets for various different densities, coordination numbers, pressures, shear-stresses and shear-rates-from a single simulation only. Due to the cylindrical symmetry, each point in $r-z$ plane, takes a certain pressure $p$, void fraction $\varepsilon$ (or density $v$ ), coordination number $C$ [28], strain rate $\dot{\gamma}$, and structural anisotropy (not discussed here). Previous simulations with dry particles were validated by experimental data and quantitative agreement was found with deviations as small as 10 per-cent $[11,12,23,29]$, concerning the center of the shear-band as function of distance from the bottom, for a recent review see Ref. [19].

Note that both time- and space-averaging are required to obtain a reasonable statistics. Furthermore, even though ringsymmetry and time-continuity are assumed for the averaging, this is not true in general, since the granular material shows strong spatial fluctuations in the deformation (nonaffine deformations) and intermittent behavior. Nevertheless, the time- and space-averages are performed as a first step to obtain continuum quantities-leaving an analysis of their fluctuations to future studies.

One important observation is that the profiles of strain rate and velocity need some time to establish, especially in their tails. The larger the local strain rate, the shorter is the time it takes the particles to establish their critical-state flow regime $[12,24]$. This means that all points close to the center of the shear-band quickly reach the steady state flow, while points farther away are not yet close to the steady flow regime. By only considering data with strain rate above a certain threshold, the critical-state shear stresses at different confining pressure and density can be obtained, representing the termination locus of the material $[11,12]$. The evolution of the shear stress towards the steady state was the subject of study in 2D [30,31] and 3D [12], but will not be addressed here. A model that connects, in particular, the evolution of stress and structure is in development and will be published elsewhere [32,33].

\section{From contacts to many-particle behavior}

The behavior of particulate media can be simulated either with the discrete element method (DEM) or with molecular dynamics (MD) [8-10,34]. Both methods share similar methodologies on the integration of the equations of motion of the particles. The difference is that MD normally involves energy conservation due to conservative forces from pair interaction potentials, while DEM handles interactions using force-displacement relations that often cannot be related to a potential due to various mechanisms of energy dissipation. MD was developed for numerical simulations of atoms and molecules [4], while DEM is more suitable for modeling geological materials and industrial powders [5]. We use the DEM approach, where the interaction forces between pairs of particles involve both normal and tangential direction and the resultant torques (as well as torques connected to rolling and torsion, which are not considered here). Particle positions, velocities and interaction forces are sufficient to integrate the equations of motion for all particles simultaneously. 
Since the exact calculation of the deformations of the particles is computationally too expensive, we assume that the particles remain spherical and can interpenetrate each other. Then we relate the normal interaction force to the overlapping length as $f^{n}=k_{n} \delta^{n}$, with a stiffness $k_{n}$ and the (interpenetration) overlap $\delta^{n}$ that stands for the contact-deformation. The tangential force $f^{t}=k_{t} \delta^{t}$ is proportional to the tangential displacement of the contact points (due to both rotations and sliding) with a stiffness $k_{t}$. The tangential force is limited by Coulomb's law for sliding $f^{t} \leq \mu f^{n}$, i.e., for $\mu=0$ one has no tangential forces at all. To account for energy dissipation, the normal and tangential degrees of freedom are also subject to viscous, velocity dependent damping forces, for more details see $[10,35]$.

\subsection{Adhesive contact model}

For fine dry particles [36], not only friction is relevant, but also adhesive contact properties due to van der Waals forces. Furthermore, due to the tiny contact area, even moderate forces can lead to plastic yield and irreversible deformation of the material in the vicinity of the contact. This complex behavior is modeled by introducing a variant of the linear hysteretic spring model, as introduced in Ref. [10] and briefly explained in the following.

The adhesive, plastic (hysteretic) force is introduced by allowing the normal stiffness constant $k_{n}$ to depend on the history of the deformation. Given the plastic stiffness $k_{1}$ and the maximal elastic stiffness $k_{2}$, the un- and re-loading stiffness $k^{*}$ interpolates between these two extremes (see below). The stiffness for un-loading increases with the previous maximal overlap, $\delta_{\max }$, reached. The overlap when the unloading force reaches zero, $\delta_{0}=\frac{k^{*}-k_{1}}{k^{*}} \delta_{\max }$, resembles the permanent plastic deformation and depends nonlinearly on the previous maximal force $f_{\max }=k_{1} \delta_{\max }$. The negative forces reached by further unloading are attractive, adhesion forces, which also increase nonlinearly with the previous maximal compression force experienced. The maximal adhesion force is given by $f_{\min }=-k_{c} \delta_{\min }$, with $\delta_{\min }=\frac{k^{*}-k_{1}}{k^{*}+k_{c}} \delta_{\max }$.

Three physical phenomena elasticity/stiffness, plasticity and adhesion are thus quantified by three material parameters $k_{2}, k_{1}$, and $k_{c}$, respectively. Plasticity disappears for $k_{1}=k_{2}$ and adhesion vanishes for $k_{c}=0$. As discussed in detail in Ref. [10], for practical reasons and since extremely high forces will lead to qualitatively different contact behavior anyway, a maximal force free overlap $\delta_{f}=$ $2 \phi_{f} a_{1} a_{2} /\left(a_{1}+a_{2}\right)$, was defined (with an empirical parameter $\phi_{f}=0.05$ ), above which $k^{*}$ does not increase anymore [10] and is set to the maximal value $k^{*}\left(\delta_{0}>\delta_{f}\right)=k_{2}$. This visco-elastic, reversible branch is referred to as "limit branch" in the following (with viscous dissipation active still). It is an over-simplification of the large-deformation regime and has some physical meaning related to multiple contacts, contact-melting, and extreme deformations, however, this is not discussed further for the sake of brevity, see Ref. [37] for details.

The magnitude of adhesion at the contacts can be quantified by the ratio of the maximal possible adhesive force and the maximal repulsive force, previously reached during loading: ${ }^{2}$

$\chi:=-\frac{f_{\min }}{f_{\max }}=\frac{k_{c}}{k_{1}} \frac{k^{*}-k_{1}}{k^{*}+k_{c}}$,

for $\delta_{0} \leq \delta_{f}$. On the limit branch one has $\chi_{f}:=\chi\left(\delta_{0} \geq\right.$ $\left.\delta_{f}\right)=\left(k_{2} / k_{1}-1\right) /\left(k_{2} / k_{c}+1\right)$ (so that $\chi_{f}=4 /\left(1+k_{2} / k_{c}\right)$ for $k_{1} / k_{2}=1 / 5$ as used for the simulations in this study). For the adhesion strengths $k_{c} / k_{2}=0,1 / 10,1 / 5,2 / 5,3 / 5$, and 1 , this leads to $\chi_{f}=0,4 / 11,2 / 3,8 / 7,3 / 2$, and 2 , respectively. The dimensionless contact property, $\chi$, quantifies the possible magnitude of cohesion relative to repulsion and can be used in the future to explain the shape of the termination locus, see Sect. 3.

\subsection{Parameters and scaling}

Note that the contact model is reasonable for fine powders [36], with (scaled) parameters given below. Before scaling, however, the parameters are arbitrary and we just use spherical particles with density $\rho=2000 \mathrm{~kg} / \mathrm{m}^{3}=2 \mathrm{~g} / \mathrm{cm}^{3}$, an average size of $a_{0}=1.1 \mathrm{~mm}$, and the width of the homogeneous size-distribution (with $a_{\min } / a_{\max }=1 / 2$ ) is $1-\mathcal{A}=$ $1-\langle a\rangle^{2} /\left\langle a^{2}\right\rangle=0.18922$. The un-scaled stiffness parameters of the model are the maximal normal stiffness $k_{2}=$ $500 \mathrm{~N} / \mathrm{m}$, the plastic stiffness $k_{1} / k_{2}=1 / 5$, and the tangential stiffness $k_{t} / k_{2}=1 / 25$. The contact friction coefficient is $\mu=0.01$, the rolling and torsion yield limits are inactive, i.e., $\mu_{r}=0.0$ and $\mu_{o}=0.0$. The normal and tangential viscosities are $\gamma_{n}=0.002 \mathrm{~kg} \mathrm{~s}^{-1}$ and $\gamma_{t} / \gamma_{n}=1 / 4$. Note that friction is chosen that artificially small in order to be able to focus on the effect of contact adhesion only.

The above values represent arbitrary numbers as used in the DEM code and, e.g., corresponding to mass-, length-, and time-units of $m_{u}=1 \mathrm{~kg}, x_{u}=1 \mathrm{~m}$, and $t_{u}=1 \mathrm{~s}$, respectively. However, as shown in Ref. [10], the dimensional numbers can be re-scaled, e.g., choosing the units $m_{u}=1 \mathrm{mg}=$ $10^{-6} \mathrm{~kg}, x_{u}=10 \mathrm{~mm}=10^{-2} \mathrm{~m}$, and $t_{u}=1 \mu \mathrm{s}=10^{-6} \mathrm{~s}$, so that the dimensional model parameters translate to $\rho=$ $2000 \mathrm{~kg} / \mathrm{m}^{3}$ (unchanged), $a_{0}=11 \mu \mathrm{m}, k_{2}=5.10^{8} \mathrm{~N} / \mathrm{m}$, and $\gamma_{n}=2.10^{-3} \mathrm{~kg} \mathrm{~s}^{-1}$ (unchanged), while the parameter ratios and other dimensionless numbers remain unaffected. In particular this order of particle size, for dry powders, is expected to display elasto-plastic, adhesive properties as implemented in the model $[10,36]$.

\footnotetext{
${ }^{2}$ Resembling $\kappa$ in Eq. (12b) in Ref. [2].
} 
In the first set of parameters (especially) size represents the original experiments of adhesionless milli-meter sized plastic particles, while the second set represents adhesive fine powders. However, since scaling between the two sets is straightforward, we use the first set of numbers in the following.

\subsection{Contact model for two particles}

Even though this paper concerns quasi-static contacts, the contact model is best visualized by plotting the contact force against overlap during the collision of two particles, see Fig. 2. At the beginning of a collision, the force increases along the $k_{1}$ branch. For large relative velocity, $v_{\text {rel }}=$ $0.4 \mathrm{~m} / \mathrm{s}$, the force reaches the $k_{2}$ branch, follows it up to high values, and then returns on the same branch during unloading until it reaches the negative $k_{c}$ branch. When it reaches the $k_{c}$ branch unloading continues along this line. For lower relative velocity, the $k_{2}$ branch is not reached and unloading takes place along a $k^{*}$ branch, where $k^{*}$ interpolates between $k_{1}$ and $k_{2}$, see Eq. (8) in Ref. [10]. Note that all collisions end with a final separation of the particles. More details and an analytic study of the sticking-related coefficient of restitution will be published elsewhere.

During a collision, in the absence of viscous forces, kinetic energy $(1 / 2) m_{\text {red }} v_{\text {rel }}^{2}$ (before collision), with reduced mass of two average size particles, $m_{\text {red }}=m_{0} / 2$, is completely trans- ferred to potential energy $(1 / 2) k_{1} \delta_{\max }^{2}$ (at maximal overlap). This leads to $\delta_{\max }=v_{\text {rel }} \sqrt{m_{\text {red }} / k_{1}}$, as an estimate for the maximal overlap reached (as long as $\delta<\delta_{f}$ ), as confirmed by the simulations in Fig. 2 .

\subsection{Model system geometry}

The geometry of the sample is described in detail in Refs. $[11,12,19,22,38]$. In brief, the assembly of spherical particles is confined by gravity between two concentric cylinders, above a split ring-shaped bottom and with a free surface at the top. The outer cylinder rotates around the symmetry-axis and the outer part of the bottom is moving together with it. The split allows that the inner part of the bottom remains at rest (like the inner cylinder). The split initiates the shearband that propagates upwards/inwards and becomes wider with increasing (height) distance from the bottom.

The simulations run for more than $50 \mathrm{~s}$ with a rotation rate $f_{o}=0.01 \mathrm{~s}^{-1}$ of the outer cylinder, with angular velocity $\Omega_{o}=2 \pi f_{o}$. For the average of the displacement, only larger times are taken into account so that the system is examined in quasi-steady state flow conditions-disregarding the transient behavior at the onset of shear. Quasi-steady state includes the possibility of very long-time relaxation effects, which can not be caught by our relatively short simulations [19].
Fig. 2 Contact force plotted against overlap for pair-collisions with relative velocity, $v_{\text {rel }}$, as given in each panel, for two particles of average radius $a=a_{0}$. The green and red lines $(\mathrm{X}$ and + symbols, respectively) represent data for the model with $(v=1)$ and without $(v=0)$ normal viscosity, respectively. The three straight lines represent the plastic branch, with slope $k_{1}$, the adhesive branch, with slope $-k_{c}$, where $k_{1} / k_{2}=k_{c} / k_{2}=1 / 5$ is used here, and the limit-branch with slope $k_{2}$ that goes through $\delta_{f}=a_{0} \phi_{f}$ at zero force. (Color figure online)
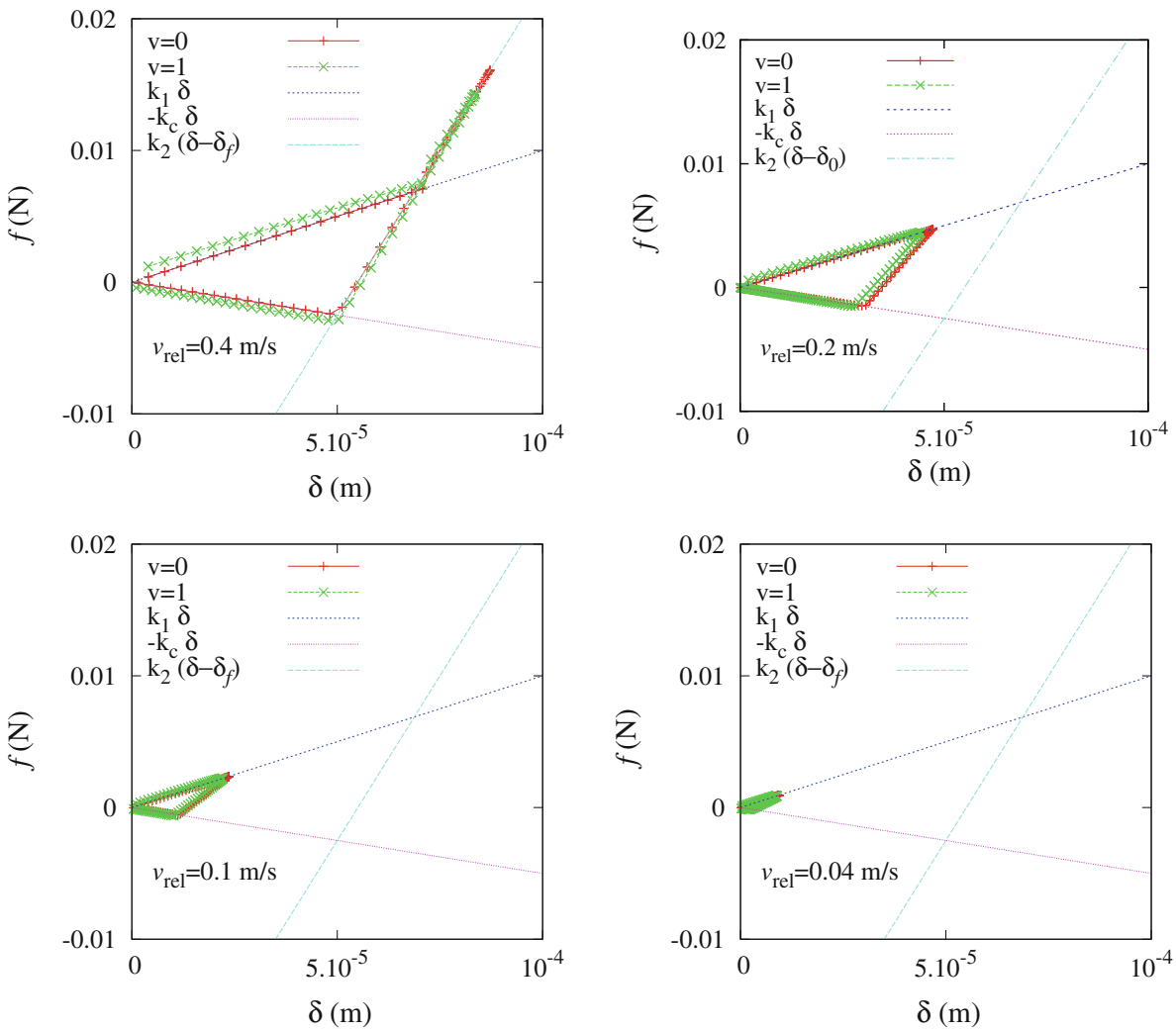
Nevertheless, we tested if the simulations are quasi-static: Three additional simulations (data not shown), two times slower, two times faster, and four times faster, confirm that the present simulation is indeed very close to quasi-static. The shear rate scales with the rotation rate $f_{o}$ as externally applied (for two times slower and faster). Only the four times faster simulation shows dynamic effects and deviates considerably from the others (when all are scaled with $f_{o}$ ).

\subsection{Contact statistics in the quasi-static situation}

The situation for the particles in the split-bottom ring shear cell is quasi-static and pair-collisions, as discussed in Sect. 2.3 are rather unlikely. Instead, the particles remain in contact for relatively long time and each contact is represented by one point in the force-overlap diagram, see Fig. 3 (where no distinction between different contact directions is made). Each point represents a contact and different symbols (colors online) represent different heights, i.e., different pressure levels. The larger the contact adhesion, the more attractive forces are active and the larger the typical overlaps are.

The particles in the cells-at a certain vertical position $z$, due to the weight of the particles above-have to sustain a certain pressure $p(z)$, where the average overlap is a (nonlinear) function of the pressure. The lower $z$, the higher the pressure, and the higher the average overlap and average forces should be. Specifically, from the simulations with contact adhesion $k_{c} / k_{2}=1 / 10$, the average overlaps are $\langle\delta\rangle \approx 0.85,1.9,3.2$, and $4.0 \times 10^{-5} \mathrm{~m}$, for pressure levels $p \approx 100,200,300$, and $400 \mathrm{~N} / \mathrm{m}^{2}$, with average forces $\langle f\rangle=0.45,0.81,1.14$, and $1.40 \mathrm{mN}$, respectively, see Fig. 3a.

For larger contact adhesion $k_{c} / k_{2}=1 / 5$, see Fig. $3 \mathrm{~b}$, the average overlaps are larger, while the average forces are not changing much. For even larger contact adhesion $k_{c} / k_{2}=$ $2 / 5$, see Fig. 3 c, the average overlaps are practically all above $\langle\delta\rangle>5 \times 10^{-5} \mathrm{~m}$, while the average forces still depend linearly on the pressure but with quite large fluctuations, probably due to the presence of more and more strong adhesive (negative) contact forces: The average positive and negative forces increase with contact adhesion strength, whereas their average does not.

Thus for strong contact adhesion, most contacts (except for very small $p$ ) drift towards and collapse around the "limit branch" of the contact model with slope $k_{2}$ (which is not based on the true physical behavior of contacts at extreme overlaps-just because this behavior is unknown to us). One possible explanation for this drift is micro-mechanical "plastic sintering": During shear, large adhesion forces become active in the tensile direction. These large attractive forces have to be compensated by even larger repulsive forces in the compressive (perpendicular) direction in order to maintain (a)

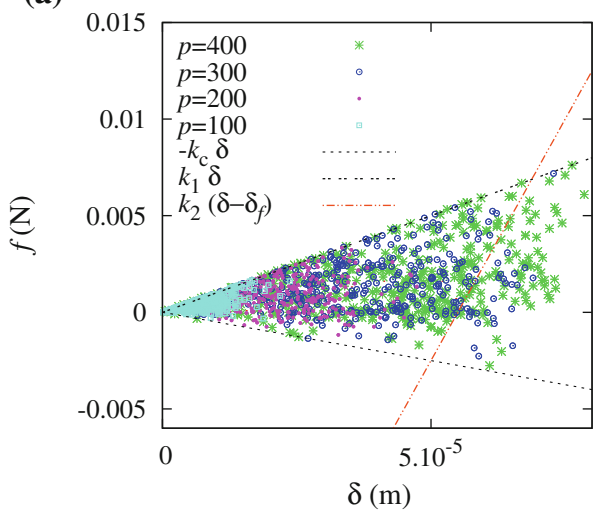

(b)

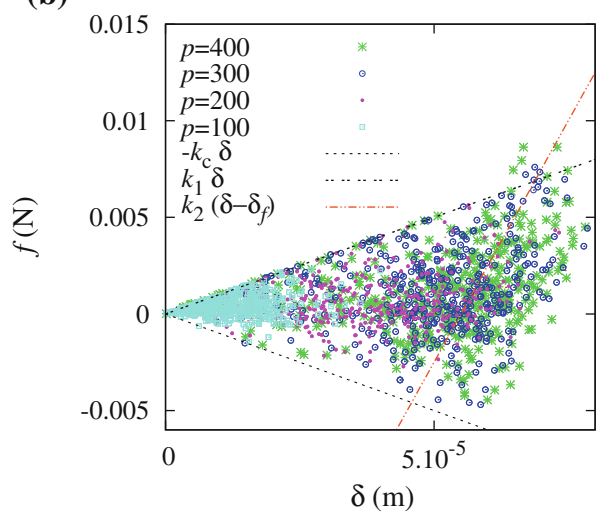

(c)

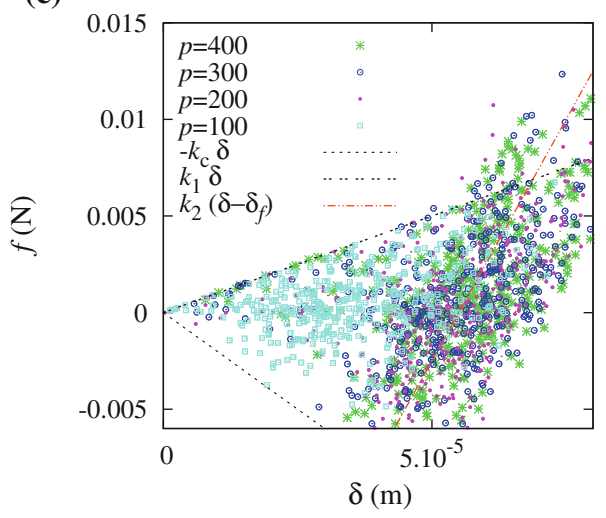

Fig. 3 Force-displacement representation of all contacts in the radial range $0.075 \mathrm{~m} \leq r \leq 0.080 \mathrm{~m}$ (red points), for different $k_{c} / k_{2}=1 / 10$ (a), 1/5 (b), and 2/5 (c). The different symbols represent a zoom into the vertical ranges $z=8 \mathrm{~mm} \pm 1 \mathrm{~mm}$ (green stars), $15 \mathrm{~mm} \pm 1 \mathrm{~mm}$ (blue circles), $22 \mathrm{~mm} \pm 1 \mathrm{~mm}$ (magenta dots), $29 \mathrm{~mm} \pm 1 \mathrm{~mm}$ (cyan squares), with approximate pressure as given in the inset. Note that the points do not collapse on the line $k_{2}\left(\delta-\delta_{f}\right)$ due to the finite width of the size distribution: pairs of larger than average particles fall out of the indicated triangle. (Color figure online)

the overall compressive pressure level. These large repulsive forces will eventually hit the plastic branch with slope $k_{1}$ and lead to increasing overlaps. ${ }^{3}$

\footnotetext{
3 A direction dependent statistics of the contact forces that distinguishes between the shear-compression and -tension directions is in progress
} 


\section{Results for varying contact adhesion}

Six realizations with the same filling height, i.e., $N \approx$ 37000 particles, are displayed in Fig. 4, both as top- and front-view without $\left(k_{c}=0\right)$ and with adhesion $\left(k_{c} / k_{2}=\right.$ $1 / 10,1 / 5,2 / 5,3 / 5$ and 1$)$. The color code indicates the displacement rate and shows (observed from the front) that the center of the shear-band moves inwards with increasing height (decreasing pressure) and with increasing contact adhesion strength. Just examined from the top (like in the original experiments), one observes that the shear-band moves inwards with increasing filling height (data not shown) and adhesion strength — and also becomes wider.

\subsection{The effect of adhesion on the shear band}

Figure 4 shows that without cohesion the shear band is narrower than with cohesion - all shear-bands being rather wide close to the free surface. Very strong cohesion makes the shear-band move so rapidly inwards that it is localized (and thus narrow) close to the bottom, see Fig. 4f.

Since the particle number is the same in all simulations, one can estimate from the bulk filling height (in front view) that the density of the more cohesive systems is smaller. Specifically, the volume fraction decays from $v \approx 0.66$ without cohesion to values as small as $v \approx 0.61$ for the strongest adhesion (in the center of the shear-band).

Interestingly, in contrast to the density, the coordination number slightly increases with increasing adhesion strength, since closed contacts are less easily opened in the presence of stronger attractive forces. The contact number density, i.e., the trace of the fabric tensor, see Refs. $[11,12,28]$, is only slightly decreasing with adhesion strength, whereas it was strongly decreasing with increasing coefficient of friction [11].

\subsection{Averaging and macroscopic results}

Since we assume translational invariance in the tangential $\phi$-direction, averaging is performed over toroidal volume elements and over many snapshots in time (typically 40-60 s), leading to fields $Q(r, z)$ as function of the radial and vertical positions. The averaging procedure was detailed for $2 \mathrm{D}$ systems, e.g., in Ref. [9], and applied to three dimensional systems $[11,12]$, so that we do not discuss the details here. Comparing the cases with different degrees of adhesive parameters in Fig. 4, we conclude that the shear-band localisation depends strongly on adhesion. To allow for a

Footnote 3 continued

with the goal to identify the mechanisms that lead to the drift and the preference for large overlaps.

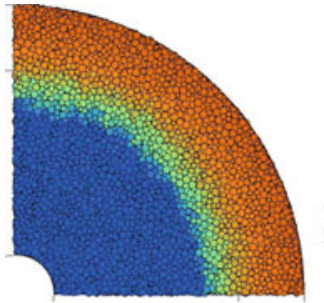

(a)
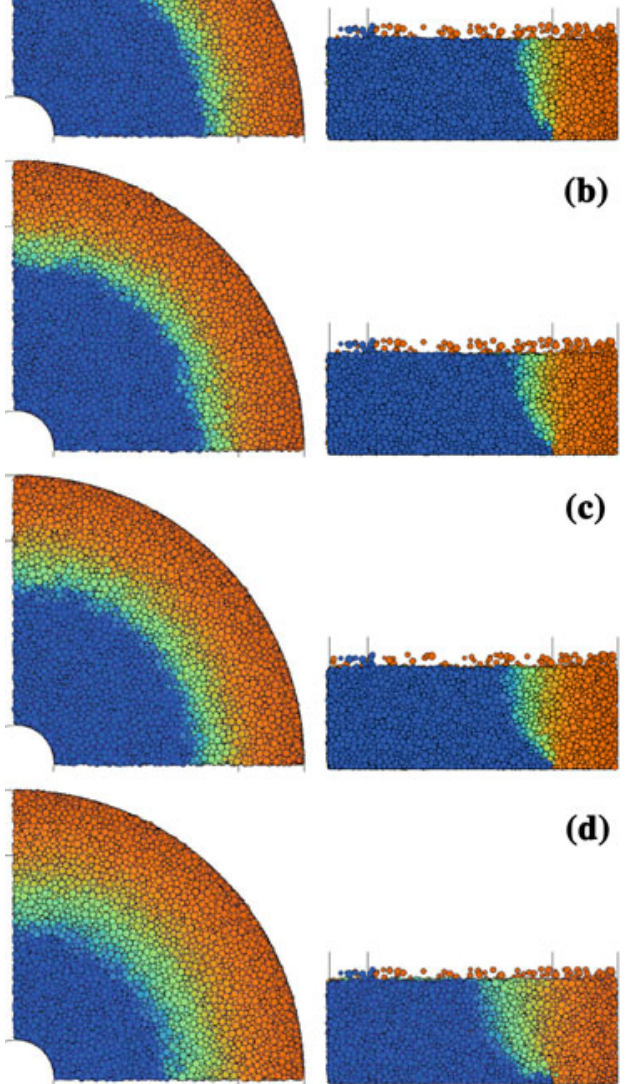

(c)
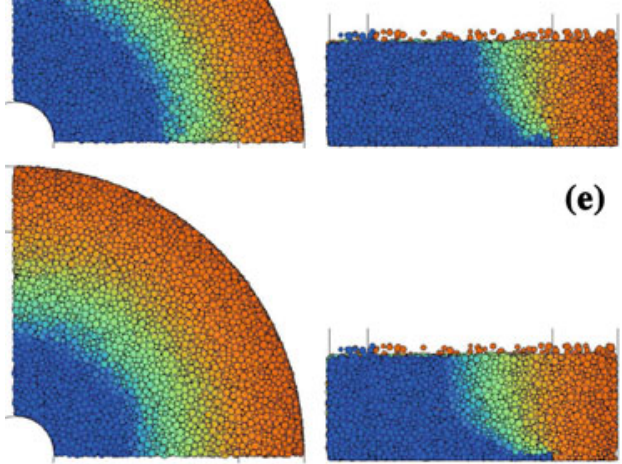

(d)
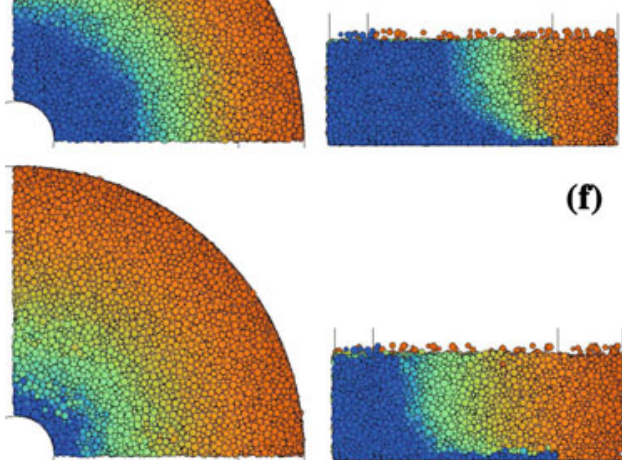

(e)

(f)

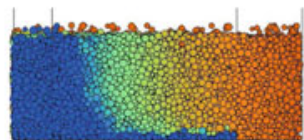

Fig. 4 Snapshots from simulations with different adhesion constants, but the same number of mobile particles $N=34518$, seen from the top (Left) and from the front (Right). The material is (a) without cohesion $k_{c} / k_{2}=0$, (b) with weak adhesion $k_{c} / k_{2}=1 / 10$, (c) with moderate adhesion $k_{c} / k_{2}=1 / 5$ and (d) $k_{c} / k_{2}=2 / 5$, and with strong adhesion (e) $k_{c} / k_{2}=3 / 5$ and (f) $k_{c} / k_{2}=1$. The colors blue, green, orange and red denote particles with displacements in tangential direction per second $r \mathrm{~d} \phi \leq 0.5 \mathrm{~mm}, r \mathrm{~d} \phi \leq 2 \mathrm{~mm}, r \mathrm{~d} \phi \leq 4 \mathrm{~mm}$, and $r \mathrm{~d} \phi>4 \mathrm{~mm}$. The displacement rate is averaged over $5 \mathrm{~s}$ intervals. Particles visible "floating" above the bulk are some of those glued to the wall. (Color figure online) 
more quantitative analysis of the width of the shear band as function of depth, fits with the universal shape function proposed in Ref. [38] can be performed for small and moderate adhesion, see Ref. [11], but will not be shown here.

A quantitative study of the averaged velocity field, and the velocity gradient derived from it, leads to the definition of the local strain rate,

$\dot{\gamma}=\frac{1}{2} \sqrt{\left(\partial v_{\phi} / \partial r-v_{\phi} / r\right)^{2}+\left(\partial v_{\phi} / \partial z\right)^{2}}$,

i.e., the shear intensity in the shear plane, as discussed in Refs. $[11,12]$. The shear plane is defined by its normal, i.e., the eigenvector of the zero eigenvalue, $\hat{e}_{0}=\hat{e}_{0}(r, z)$, of the symmetrized strain rate tensor, which — due to the cylindrical geometry-lies in the $r-z$-plane. Thus, the orientation of the shear plane can be described by a single angle $[11,12]$. The other two eigenvectors lie in the shear plane and are rotated (around $\hat{e}_{0}$ ) by $\pm 45^{0}$ out of the $r-z$-plane.

Furthermore, one can determine the components of the (static) stress tensor as $\sigma_{\alpha \beta}=\frac{1}{V} \sum_{c \in V} f_{\alpha} l_{\beta}$, with the components of the contact forces $f_{\alpha}$ and branch vectors $l_{\beta}$ that connect the centers of mass of the particles with the contact points. The sum extends over all contacts within or close to the averaging volume, weighted according to their vicinity. Note that we disregard the (very small) tangential forces here for the sake of simplicity. The shear stress is defined in analogy to the shear strain, as proposed in Ref. [39], so that: $|\tau|=\sqrt{\sigma_{r \phi}^{2}+\sigma_{z \phi}^{2}}$. The other components of stress as well as its eigenvalues and eigenvectors (relative to those of the strain tensor) will be discussed elsewhere.

Remarkably, for non-cohesive materials the macroscopic critical state coefficient of friction, $\left|\tau^{*}\right| / p \approx \mu_{m}^{*}$, is well defined (data not shown, see Refs. $[11,12]$ ), i.e. the slope of the shear stress-pressure curve is almost constant for practically all averaging volumina with strain rates larger than some threshold value. In other words, if the dimensionless shear length [12] $l_{\gamma} \approx t_{a v} \dot{\gamma} \gg 1$, with averaging time $t_{a v}$, clearly exceeds one particle diameter, the shear deformation can be assumed to be fully established-resembling the concept of a critical flow regime. For the present data-set, with averaging times $t_{a v} \approx 10 \mathrm{~s}$, we observe that $\dot{\gamma} \geq \dot{\gamma}_{c}$, with $\dot{\gamma}_{c} \approx 0.08 \mathrm{~s}^{-1}$ is the shear-rate above which the shearbands are close to fully established. However, this (and the ongoing drift-see above) makes the data for large cohesion unreliable, especially close to the surface. This is due to the very wide shear-bands, the shear rates are rather low for $k_{c} / k_{2} \geq 2 / 5$. Those data require much longer simulation times in order to make sure that the critical-state regime is really established.

\subsection{Shape of the termination locus}

When elasto-plasticity and contact adhesion is included in the model, a nonlinear termination locus is obtained with a peculiar pressure dependence. This nonlinearity becomes apparent when we plot the shear stress against pressure for different coefficients of adhesion, as shown in Fig. 5. The main effect of contact adhesion is that it increases the strength of the material under large confining stress, but not for small $p$, i.e., close to the surface. For very weak adhesion the strength is given by the linear relation between shear stress and pressure like for non-adhesive material. In Fig. 5, the termination locus is well fitted by the function

$\left|\tau^{*}\right|=\mu_{m}^{*} p+c_{2}\left(\frac{p}{p_{f}}\right)^{2}$

with $\mu_{m}^{*}=0.15, p_{f}=200 \mathrm{Nm}^{-2}$, and (a) $c_{2}=4$, (b) $c_{2}=15$, (c) $c_{2}=68 \mathrm{Nm}^{-2}$, as indicated by the dashed lines. (We confirmed that $p \approx \sigma^{*}$ with $p$ slightly larger. The small value of $c_{2}>0$ for cohesionless material is probably due to the rather small ratio $k_{1} / k_{2}=1 / 5$, which is different from the previously reported data for which there was no plastic regime and no cohesion [11].)

For high confining stress $p>p_{f}$, the shear stress increases much less than predicted by the power law. One rather has a classical cohesion $\left|\tau^{*}\right|=\mu_{m}^{*} p+c$, with $c=\chi_{f} c_{f}, c_{f} \approx 150 \mathrm{Nm}^{-2}$, and $\chi_{f}$ given below Eq. (3). While a single value of $c_{f}$, together with the analytical expression for $\chi_{f}$, fits the data for large confining pressure (for contacts mostly on the limit branch), the study of the parameters $c_{2}$ and their relation to $\chi$ is in progress.

The microscopic reason for the nonlinearity of the termination locus is the nonlinear contact model: The contacts feel practically no adhesion forces for very small experienced pressure (close to the free surface). Larger adhesion forces can be active for higher pressures in the bulk of the material, due to a nonlinear increase of the contact adhesion with increasing pressure in the plastic regime of the contact model for overlaps $\delta<\delta_{f}$, see Fig. 3b. For extremely high confining stress $p>p_{f}$, the majority of contacts resides on the visco-elastic limit branch of the contact model, where the maximal possible adhesion force is constant and the overlaps are $\delta \approx \delta_{f}$, see Fig. 3c. Thus the micro-mechanical mechanisms involve not only elasto-plastic deformation of the contact and therefore adhesion, but also "plastic sintering" (see Sect. 2.5) during re-loading cycles under continuous shear.

\section{Conclusions}

Simulations of a split-bottom Couette ring shear cell with dry granular materials show perfect qualitative and good quantitative agreement with experiments. The effect of 

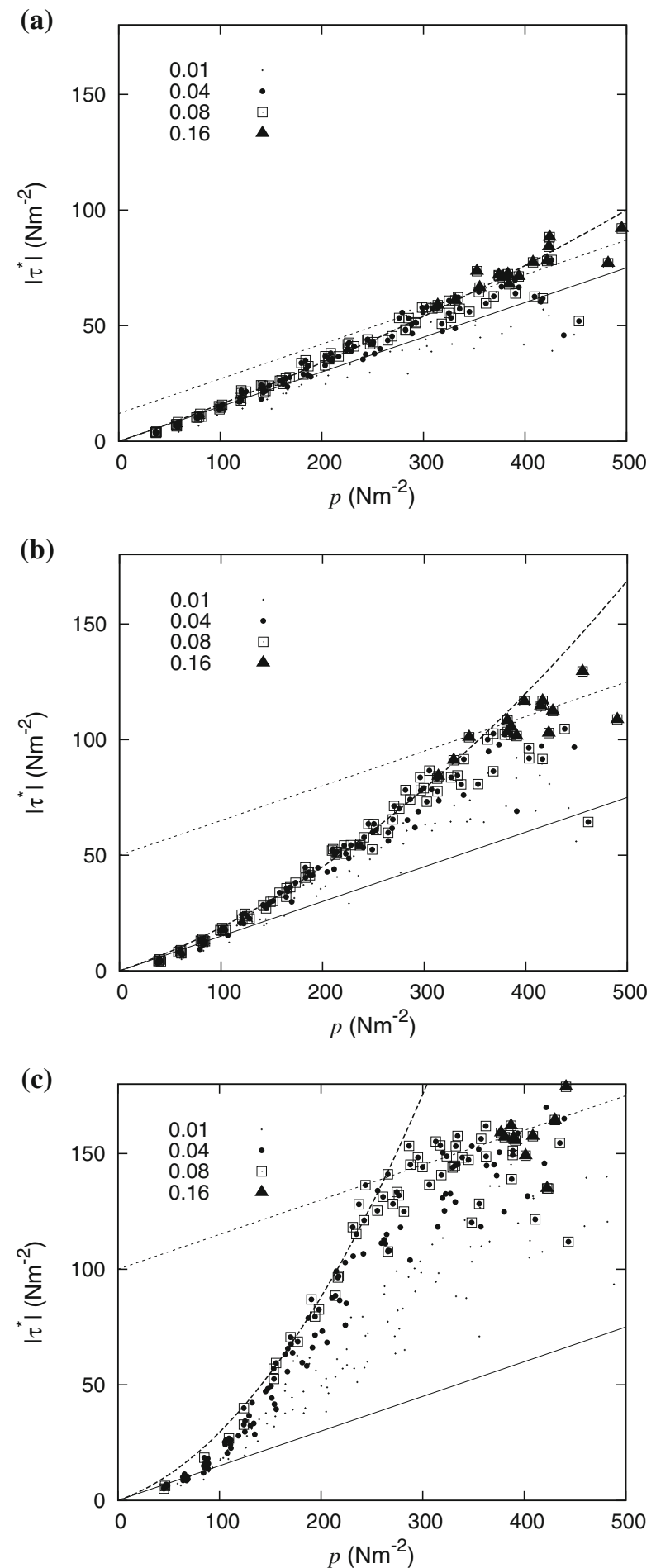

Fig. 5 Shear stress $|\tau|$ plotted against pressure $p$ for three different adhesive parameters: $k_{c} / k_{2}=0(\mathbf{a}), k_{c} / k_{2}=1 / 10(\mathbf{b})$, and $k_{c} / k_{2}=$ $1 / 5$ (c). The magnitude of the strain rate, i.e., $\dot{\gamma} \geq \dot{\gamma}_{i}$, with the $\dot{\gamma}_{i}$ given in the insets in units of $\mathrm{s}^{-1}$. The solid line represents the function $\tau_{\max }=\mu_{m}^{*} p$, where $\mu_{m}^{*}=0.15$ was used here, while the dashed lines are the parabolic fits in Eq. (5), as discussed in the main text. The dotted lines represent $\tau_{\max }+c_{f} \chi_{f}$, with one reference macroscopic cohesion stress $c_{f} \approx 150 \mathrm{Nm}^{-2}$ for $1 / 10 \leq k_{c} / k_{2} \leq 2 / 5$. The dotted line in (a), where $\chi_{f}=0$, is just an arbitrary fit $\tau_{\max }+12 \mathrm{Nm}^{-2}$ friction was studied recently, so that in this study the effect of contact adhesion was examined in some detail.

Like for particles without adhesion, the shear-band is triggered by the split in the bottom and then its center moves inwards with increasing height (decreasing pressure) due to the ring-geometry. It moves in faster/further and becomes wider with increasing contact adhesion. For very strong contact adhesion, the shear-band localizes close to the bottom wall.

The termination locus, i.e., the maximal shear stress, $\left|\tau^{*}\right|$ in critical-state flow, also called critical-state yield stress, when plotted against pressure-for those parts of the system that have experienced considerable shear (displacement)is almost linear in the absence of adhesion, corresponding to a linear Mohr-Coulomb type critical-state line (termination locus) with slope (macroscopic critical-state coefficient of friction) $\mu_{m}^{*}=\tan \Delta$, increasing with microscopic contact friction (data not shown). A strong nonlinearity of the termination locus emerges as a consequence of the strong adhesive forces that increase nonlinearly with the confining pressure: Attractive forces are very weak for low pressure and increase considerably for larger pressure in the presence of strong contact adhesion. Saturation is observed, since the contact adhesion force cannot grow beyond a certain threshold (by construction). Therefore, due to this nonlinearity, the definition of a macroscopic cohesion (shear stress at zero normal stress) becomes questionable for low pressure levels, but is meaningful at higher confining pressure.

The interesting phenomenology is due to the history dependent contact model: Particles and contacts that have experienced large pressure can provide much larger adhesive forces than others, which have not been compressed a lot previously. Therefore, at the top (free surface with low pressure) the yield stress and resistance to shear flow is much lower than deep inside the sample (high pressure), where due to the continuous shear and contact re-loading, contacts have developed towards large adhesion forces.

The physical origin of the nonlinearity in the contact model is the permanent deformation at contact, which leads to a larger contact surface area and therefore to a stronger pull-off force (due to van der Waals forces). As final remark, we note that the model contains two unphysical simplification: (i) At extreme overlaps, a linear limit force model is used with a constant maximal adhesion, and (ii) the longer ranged van der Waals adhesion is neglected and only the contact adhesion is considered. Future studies with the longer range (non-contact) term will show whether this can lead to a more linear, convex yield (termination) locus. In real systems of dry, adhesive powders, the longer ranged adhesion will provide some bulk cohesion, since-as shown in this study - the contact adhesion alone is not effective at small confining pressure. 
Besides the study of several open issues, as raised in this paper, future research will involve different shear rates, different coefficients of friction, rolling- and torsion-resistance as well as non-spherical shapes. Furthermore, the microscopic contact network and force statistics in the presence of adhesion has to be better understood as well as the interplay between structure, stress and strain-with the goal to define objective constitutive laws based on the micro-mechanics. Finally, the present numerical results should be calibrated and validated by experiments.

Acknowledgments Helpful discussions with V. Magnanimo and A. Singh are acknowledged as well as the helpful criticism of both referees of our paper and the financial support of the Deutsche Forschungsgemeinschaft (DFG), the Stichting voor Fundamenteel Onderzoek der Materie (FOM), financially supported by the Nederlandse Organisatie voor Wetenschappelijk Onderzoek (NWO). F. Alonso-Marroquín is supported by the Australian Research Council and the Australian Academy of Sciences.

\section{References}

1. Williams, J.C., Birks, A.H.: The comparison of the failure measurements with theory. Powder Technol. 1, 199-206 (1967)

2. Molerus, O.: Theory of yield of cohesive powders. Powder Technol. 12, 259-275 (1975)

3. Nedderman, R.M.: Statics and kinematics of granular materials. Cambridge University Press, Cambridge (1992)

4. Allen, M.P., Tildesley, D.J.: Computer Simulation of Liquids. Oxford University Press, Oxford (1987)

5. Cundall, P.A.: A computer model for simulating progressive, largescale movements in blocky rock systems. In: Proc. Symp. Int. Rock Mech. Vol. 2(8), Nancy, (1971)

6. Alonso-Marroquin, F., Luding, S., Herrmann, H.J., Vardoulakis, I.: Role of anisotropy in the elastoplastic response of a polygonal packing. Phys. Rev. E 71, 051304-1-051304-18 (2005)

7. Bagi, K.: Microstructural stress tensor of granular assemblies with volume forces. J. Appl. Mech. 66, 934-936 (1999)

8. Vermeer, P.A., Diebels, S., Ehlers, W., Herrmann, H.J., Luding, S., Ramm, E. (eds.): Continuous and Discontinuous Modelling of Cohesive Frictional Materials. Lecture Notes in Physics, vol. 568. Springer, Berlin, (2001)

9. Lätzel, M., Luding, S., Herrmann, H.J.: Macroscopic material properties from quasi-static, microscopic simulations of a twodimensional shear-cell. Granul. Matter 2(3), 123-135 (e-print cond-mat/0003180) (2000)

10. Luding, S.: Cohesive frictional powders: contact models for tension. Granul. Matter 10, 235-246 (2008)

11. Luding, S.: The effect of friction on wide shear bands. Part. Sci. Technol. 26(1), 33-42 (2008)

12. Luding, S.: Constitutive relations for the shear band evolution in granular matter under large strain. Particuology 6(6), 501505 (2008)

13. Schwedes, J.: Review on testers for measuring flow properties of bulk solids. Granul. Matter 5(1), 1-45 (2003)

14. Atkinson, J.H., Brandsby, P.L.: Mechanics of Soils. McGraw-Hill Book Co., London (1978)

15. Rottler, J., Robbins, M.O.: Yield conditions for deformations of amorphous polymer glasses. Phys. Rev. E 64, 051801 (2001)
16. Coussot, P., Nguyen, Q.D., Huynh, H.T., Bonn, D.: Avalanche behavior in yield stress fluids. Phys. Rev. Lett. 88(17), 175501 (2002)

17. Rottler, J., Robbins, M.O.: Unified description of aging and rate effects in yield of glassy solids. Phys. Rev. Lett. 95, 225504 (2005)

18. Beruto, D.T., Lagazzo, A., Botter, R., Grillo, R.: Yield stress measurements and microstructure of colloidal kaolin powders clusterized and dispersed in different liquids. Particuology 7, 438444 (2009)

19. Dijksman, J.A., van Hecke, M.: Granular flows in split-bottom geometries. Soft Matter 6, 2901-2907 (2010)

20. Bonn, D., Tanaka, H., Coussot, P., Meunier, J.: Ageing, shear rejuvenation and avalanches in soft glassy materials. J. Phys. Condens. Matter 16(42), S4987 (2004)

21. Lätzel, M., Luding, S., Herrmann, H.J., Howell, D.W., Behringer, R.P.: Comparing simulation and experiment of a $2 \mathrm{~d}$ granular couette shear device. Eur. Phys. J. E 11(4), 325-333 (2003)

22. Fenistein, D., van de Meent, J.W., van Hecke, M.: Universal and wide shear zones in granular bulk flow. Phys. Rev. Lett. 92, 094301 (e-print cond-mat/0310409) (2004)

23. Luding, S., Alonso-Marroquin, F.: How to get the yield locus of an adhesive powder from a single numerical experiment. In: Wypych, P. (ed.) 6th International Conference for Conveying and Handling of Particulate Solids, CHOPS09 Proceedings (3-7 August 2009, Brisbane, QLD, Australia), ISBN: 978-0858259065, pp. 472-477. Engineers Australia, 11 National Circuit, Barton ACT 2600 (2009)

24. Ries, A., Wolf, D.E., Unger, T.: Shear zones in granular media: three-dimensional contact dynamics simulations. Phys. Rev. E 76, 051301 (2007)

25. Jop, P.: Hydrodynamic modeling of granular flows in a modified Couette cell. Phys. Rev. E 77, 032301 (2008)

26. Jagla, E.A.: Finite width of quasi-static shear bands. Phys. Rev. E 78, 026105 (2008)

27. Fan, Y., Hill, K.M.: Shear driven segregation of dense granular mixtures in a split-bottom cell. Phys. Rev. E 81, 041303 (2010)

28. Göncü, F., Duran, O., Luding, S.: Constitutive relations for the isotropic deformation of frictionless packings of polydisperse spheres. C. R. Mecanique 338, 570-586 (2010)

29. Luding, S.: Objective constitutive relations from DEM. In: Grabe, J. (ed.) Seehäfen für Containerschiffe zukünftiger Generationen, pp. 173-182. TUHH, Germany, GB (2008)

30. Luding, S.: Shear flow modeling of cohesive and frictional fine powder. Powder Technol. 158, 45-50 (2005)

31. Luding, S.: Anisotropy in cohesive, frictional granular media. J. Phys. Condens. Matter 17, S2623-S2640 (2005)

32. Luding, S., Perdahcioglu, S.: A local constitutive model with anisotropy for various homogeneous $2 \mathrm{~d}$ biaxial deformation modes. CIT, (2011); submitted

33. Magnanimo, V., Luding, S.: A local constitutive model with anisotropy for ratcheting under $2 \mathrm{~d}$ biaxial isobaric deformation. Granul. Matter (2011); submitted

34. Luding, S.: Micro-macro transition for anisotropic, frictional granular packings. Int. J. Sol. Struct. 41, 5821-5836 (2004)

35. Luding, S.: Collisions \& contacts between two particles. In: Herrmann, H.J., Hovi, J.-P., Luding, S. (eds.) Physics of Dry Granular Media-NATO ASI Series E350, pp. 285 Kluwer, Dordrecht (1998)

36. Tomas, J.: Fundamentals of cohesive powder consolidation and flow. Granul. Matter 6(2/3), 75-86 (2004)

37. Luding, S., Manetsberger, K., Muellers, J.: A discrete model for long time sintering. J. Mech. Phys. Solids 53(2), 455-491 (2005)

38. Fenistein, D., van Hecke, M.: Kinematics-wide shear zones in granular bulk flow. Nature 425(6955), 256 (2003)

39. Depken, M., van Saarloos, W., van Hecke, M.: Continuum approach to wide shear zones in quasistatic granular matter. Phys. Rev. E 73, 031302 (2006) 